\title{
Rayleigh Instability of Plane-parallel Liquid Flows
}

\author{
B. M. Dakhel \\ General Required Courses Department, Jeddah Community College \\ King Abdulaziz University, Jeddah, Saudi Arabia
}

Received: December 16, 2011

Accepted: January 5, 2012 Published: February 1, 2012

doi:10.5539/mas.v6n2p81

URL: http://dx.doi.org/10.5539/mas.v6n2p81

\begin{abstract}
Studying in this paper the stability of plane-parallel flows of an ordinary liquid can be naturally translated into the language of the theory of hydrodynamic resonances. Thus, resonant absorption of oscillations induces stability of the flows of an ideal liquid having a velocity profile without inflection points (Rayleigh theorem), while resonant emission leads to Rayleigh instability in the presence of an inflection point. The flow velocity profile has an inflection point. Thus, the presence of inflection points is a necessary condition for instability. If, however, the velocity profile has inflection points, the flow is stable (Rayleigh's theorem). Note that the sign of the jump depends on whether the neutral oscillations are regarded as the limiting cases of growing $(\operatorname{Im} \omega>0)$ or damped $(\operatorname{Im} \omega<0)$ oscillations.
\end{abstract}

Keywords: Rayleigh Instability, Plane-Parallel Liquid Flows

\section{Introduction}

Rayleigh has established in 1880 (Rayleigh, 1880), that plane-parallel liquid flows (Barston, 1991) of an ideal liquid, with velocity profiles that have no inflection points, are stable Rayleigh's theorem (Rayleigh, 1880).

Rayleigh instability (Khenner, et al., 1999; Wolf, 1970a, 1997b; Kumar, et al., 1994) occurs when a heavy fluid is supported by a lighter fluid. Any perturbation of the interface grows and leads to spikes of the heavier fluid penetrating into the lighter one.

It is known that vertical vibrations can lead both to the parametric excitation of waves at the interface and to the suppression of the Rayleigh-Taylor instability (Benjamin, et al., 1954; Miller, et al., 1983; Kumar, et al., 1994; Wolf, 1970), while the effects due to horizontal vibrations have been studied less. In experimental works by (Bezdeneznykh, et al., 1984; Piriz, et al., 2010) for a long horizontal reservoir filled with two immiscible viscous fluids, an interesting phenomenon was found at the interface: the horizontal vibrations lead to the formation of a steady relief. This formation mechanism has a threshold nature; it is noteworthy that such a wavy relief appears on the interface only if the densities of the two fluids are close enough, i.e. it does not appear for the liquid/gas interface.

Recently, (Dou, 2002; Kuznetsov, et al., 2011) proposed a new mechanism for flow instability and turbulent transition in parallel shear flows. In this paper, based on the previous work (Dou, 2002; Kuznetsov, et al., 2011), it is demonstrated that the stability of plane-parallel flows of an ordinary liquid can be naturally translated into the language of the theory of hydrodynamic resonances.

\section{Basic Equations}

Rayleigh's theorem deals with oscillations of an ideal liquid. The result of the theorem is valid for the flows of a real liquid as sufficiently large Reynolds numbers, when the influence of viscosity can be neglected. In this approximation, the equation of motion takes the following form

$$
\frac{d \vec{V}}{d t}=-\frac{\nabla p}{\rho}
$$

Where $p$ is the pressure and $\rho$ is the density of the medium.

If the liquid is incompressible, the velocity $\vec{V}$ must also satisfy the equation

$\nabla \cdot \vec{V}=0$.

In the $x y$-plane the last equation will be satisfied if $\vec{V}=(\partial \psi / \partial y ;-\partial \psi / \partial x)$, where $\psi$ is the flow function. 
Taking the $z$-component of the curl of (1) and expressing the velocity in terms of the flow current function, then

$$
\frac{d}{d t} \Delta \psi=0
$$

This equation is called the law of conservation of the velocity curl in an ideal incompressible liquid $\left(\nabla \times \vec{V}=z_{0} \Delta \psi\right)$.

Linearizing (2) in terms of small perturbations, then becomes

$$
\left(\frac{\partial}{\partial t}+V_{0}(x) \frac{\partial}{\partial y}\right) \Delta \psi_{1}-\frac{d^{2} V_{0}}{d t^{2}} \frac{\partial \psi_{1}}{\partial y}=0
$$

It is assumed here that a small perturbation characterized by $\psi_{1}$ is applied to a stationary flow having a velocity directed along $y$-axis and varying along $x$-axis.

By using the following perturbation

$$
\psi_{1}(\vec{r}, t)=\psi_{1}(x) e^{-i(\omega t-k y)}
$$

Then we get

$$
\psi_{1}^{\prime \prime}+\left(\frac{k V_{0}^{\prime \prime}}{\omega-k V_{0}}-k^{2}\right) \psi_{1}=0
$$

This equation is called Rayleigh equation.

We shall follow (Lin, 1955). We multiply (4) by $\psi_{1}^{*}$ and subtract from the product of the complex conjugate

$$
\frac{d}{d x}\left(\psi_{1}^{*} \frac{d \psi_{1}}{d x}-\psi_{1} \frac{d \psi_{1}^{*}}{d x}\right)=2 i \frac{\operatorname{Im} \omega k V_{0}^{\prime \prime}}{\left|\omega-k V_{0}\right|^{2}}\left|\psi_{1}\right|^{2}
$$

Integrating (5) from one boundary of the flow to the other

$$
W||_{x_{1}}^{x_{2}}=k \operatorname{Im} \omega \int_{x_{1}}^{x_{2}} \frac{k V_{0}^{\prime \prime}}{\left|\omega-k V_{0}\right|^{2}}\left|\psi_{1}\right|^{2} d x
$$

We have introduced in (6) the real quantity $W=(i k / 2)\left[\psi_{1}\left(d \psi_{1}^{*} / d x\right)-\psi_{1}^{*}\left(d \psi_{1} / d x\right)\right]$. It defines the so-called Reynolds stress

$$
\widetilde{W}_{T}=\rho W=-\rho \operatorname{Re}\left(V_{x_{1}} V_{y_{1}}^{*}\right)
$$

in the oscillations.

Equation (5), which is the differential equivalent of (6), shows that at $\operatorname{Im} \omega=0$ the function $W$ is constant in the intervals and undergoes a jump at the resonant point. Note that the sign of the jump depends on whether the neutral oscillations are regarded as the limiting cases of growing $(\operatorname{Im} \omega>0)$ or damped $(\operatorname{Im} \omega<0)$ oscillations.

The normal velocity component $V_{x_{1}}=i k \psi_{1}$ and with it the left-hand side of (6) vanish on the solid walls that bound the flow. For $\operatorname{Im} \omega \neq 0$, however, the right-hand side of this equation can vanish only if $V_{0}^{\prime \prime}$ reverses sign in the interval $\left(x_{1}, x_{2}\right)$, i.e., the flow velocity profile has an inflection point. Thus, the presence of inflection points is a necessary condition for instability. If, however, the velocity profile has now inflection points, the flow is stable (Rayleigh's theorem).

\section{Conclusions}

In this paper we study the basic laws determining the stability of plane-parallel flows of an ordinary liquid can be naturally translated into the language of the theory of hydrodynamic resonances. Thus, resonant absorption of oscillations induces stability of the flows of an ideal liquid having a velocity profile without inflection points (Rayleigh theorem), while resonant emission leads to Rayleigh instability in the presence of an inflection point.

Note that at first glance Equation (6) applies equally well to growing $(\operatorname{Im} \omega>0)$ and attenuating $(\operatorname{Im} \omega<0)$ oscillations. One would therefore conclude from (6) that if some natural oscillations are in fact 
analysis of damped oscillations $(\operatorname{Im} \omega<0)$ calls for taking into account the viscosity, no matter how low, of the liquid.

Therefore all the conclusions concerning oscillations with $\operatorname{Im} \omega<0$, based on an analysis of the equation of an ideal liquid, are, generally speaking, incorrect.

\section{References}

Barston, E. M. (1991). On the linear stability of inviscid incompressible plane parallel flow. J. Fluid Mech., 233, 157-163. http://dx.doi.rog/10.1017/S0022112091000435

Benjamin, T. B., \& Ursell, F. (1954). The Stability of the Plane Free Surface of a Liquid in Vertical Periodic Motion. P. Roy. Soc. Lond. A., 225, 505-515. http://dx.doi.rog/10.1098/rspa.1954.0218

Bezdenezhnykh, N. A., Briskman, V. A., Lyubimov, D. V., Cherepanov, A. A., \& Sharov, M. T. (1984). Control of the fluid interface stability by vibration, electric and magnetic fields, in: III All-Union Seminar on Hydromechanics and Heat/Mass Transfer in Microgravity, Chernogolovka, pp. 18-20 (in Russian).

Dou, H S. (2002). Energy gradient theory of hydrodynamic instability, Technical Report of National University of Singapore, also submitted, 2003.

Khenner, M. V., Lyubimov, D. V., Belozerova, T. S., \& Roux, B. (1999). Stability of plane-parallel vibrational flow in a two-layer system. Eur. J. Mech.B/ Fluids, 18, 1085-1101. http://dx.doi.org/10.1016/S0997-7546(99)00143-0

Kumar, K., \& Tuckerman, L. S. (1994). Parametric instability of the interface between two fluids. J. Fluid Mech., 279, 49-68. http://dx.doi.rog/10.1017/S0022112094003812

Kuznetsov, S. V., \& Nafasov, A. E. (2011). Horizontal Acoustic Barriers for Protection from Seismic Waves. Advances in Acoustics and Vibration, 2011, 150310-150318. http://dx.doi.rog/10.1155/2011/150310

Lin, C. C. (1955). Theory of hydrodynamic stability, Cambridge Univ. Press.

Miller, D. L., \& Nyborg, W. L. (1983). Theoretical investigation of the response of gas-filled micropores and cavitation nuclei to ultrasound. J. Acoust. Soc. Am., 73, 1537-1544. http://dx.doi.org/10.1121/1.389415

Or, A. C. (1997). Finite-wavelength instability in a horizontal liquid layer on an oscillating plane. J. Fluid Mech., 335, 213-232. http://dx.doi.rog/10.1017/S0022112062001366

Piriz, A. R., Prieto, G. R., Díaz, I. M., López Cela, J. J., \& Tahir, N. A. (2010). Dynamic stabilization of Rayleigh-Taylor instability in Newtonian fluids. Phys. Rev. E., 82, 026317-026328. http://dx.doi.org/10.1103/PhysRevE.82.026317

Rayleigh, L. (1880). On the stability or instability of certain fluid motions. Proc. Lond. Maths. Soc., 11, 57-72. http://dx.doi.rog/10.1112/plms/s1-11.1.57

Wolf, G. H. (1970). Dynamic Stabilization of the Interchange Instability of a Liquid-Gas Interface. Phys. Rev. Lett., 24 (9), 444-446. http://dx.doi.rog/10.1103/PhysRevLett.24.444 